\title{
COVID-19, host response treatment, and the need for political leadership
}

\author{
David S. Fedson ${ }^{1}$
}

Accepted: 28 October 2020 / Published online: 2 December 2020

(c) Springer Nature Limited 2020

\begin{abstract}
Health officials and scientists have warned that we face the threat of a potentially devastating influenza pandemic. Instead, we are now in the midst of a global coronavirus (COVID-19) pandemic. National and international pandemic preparedness plans have focused on developing vaccines and antiviral treatments. Another way to confront the COVID-19 pandemic (and future pandemics) might be to treat patients with inexpensive and widely available generic drugs that target the host response to infection, not the virus itself. The feasibility of this idea was tested during the Ebola outbreak in West Africa in 2014. This experience should inform our approach to treating COVID-19 patients. It could also save lives during outbreaks of other emerging infectious diseases and episodes of everyday acute critical illness. If this "bottom up" syndromic approach to treating acute critical illness were shown to be effective, it could have a dramatic impact on health, equity and security throughout the world.
\end{abstract}

\section{Highlights:}

- Uncertainty about the outcome of COVID-19 is driving the social, economic and political distress associated with the pandemic.

- Treating the host response to COVID-19 with inexpensive and widely available generic drugs might save lives and mitigate this distress.

- Undertaking research on this idea will require political leadership.

Keywords COVID-19 $\cdot$ Host response $\cdot$ Generic drugs $\cdot$ ACE2 $\cdot$ Angiotensin receptor blockers $\cdot$ Statins

David S. Fedson

davidsfedson@gmail.com

157 chemin du Lavoir, 01630 Sergy Haut, France 


\section{Introduction}

The 'sound' banker ... is not one who sees danger and avoids it, but one who, when he is ruined, is ruined in a conventional and orthodox way along with his fellows so that no one can really blame him.

John Maynard Keynes

"The Consequences to the Banks of the Collapse of Money Values" 1931

In this quote, John Maynard Keynes-one of the most influential 20th-century economists - talks about assigning responsibility for the actions of bankers and the reluctance or unwillingness of societies to hold them accountable for what they do or do not do. In the US election of 2020, voters decided to hold the Trump administration accountable for its failure to mount an effective response to the COVID-19 pandemic. Voters were not called upon to decide whether elite scientists and institutions should be held accountable for failing to undertake research on the idea that host response treatment might offer the only practical way to reduce COVID-19 mortality. The consequences of the failure to take this idea seriously were self-evident in the influenza pandemic in 2009 and during the Ebola outbreaks in West Africa in 2014 and in the DRC in 2019. For whatever reasons, the consequences of this failure continue to this day.

More than 15 years ago, cardiologists learned that among patients with acute myocardial infarction (an inflammatory disease), those who had been taking statins as outpatients and continued taking them or started on statins after hospitalization had better survival than those who were not treated [1]. Importantly, those who had been taking statins as outpatients but discontinued them after hospitalization had increased mortality. Other investigators later found that pneumonia patients who had been taking statins, angiotensin receptor blockers (ARBs) or angiotensin-converting enzyme inhibitors (ACEIs) had improved survival [2]. An observational study by the same investigators showed that outpatient treatment with a statin/ARB combination also greatly improved pneumonia survival compared with single drug treatment [2; see Table 1]. In 2014, an unconventional and poorly documented experience with Ebola patients in Sierra Leone suggested that combination statin/ARB treatment led to "remarkable improvement" in patient survival [2]. Taken together, these studies suggest that treatments targeting the host response with widely available, inexpensive generic drugs could improve survival in patients with emerging infections and other acute inflammatory illnesses. 
Table 1 A research agenda for treating the host response to COVID-19

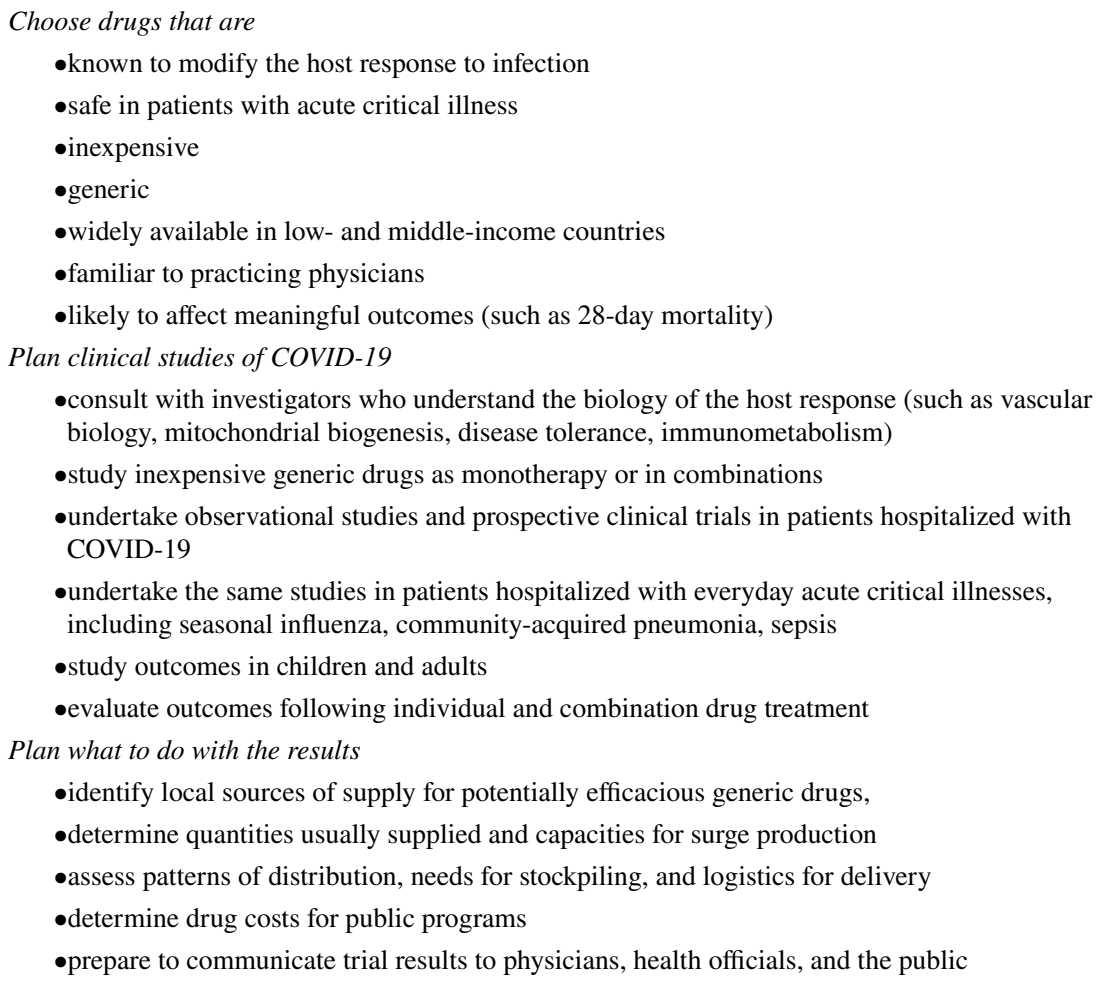

\section{COVID-19: the disease}

The respiratory disease (COVID-19) that emerged in China and has spread throughout the world is caused by a coronavirus (SARS-CoV-2). Most infections cause only mild to moderate illness and many have been asymptomatic. Case fatality rates in hospitalized adults have been greater than those seen with seasonal influenza, but there have been few hospitalizations or deaths among children. Travel bans and physical distancing measures implemented by governments might mitigate some of the devastating worldwide social, political and economic distress COVID-19 has caused; by themselves they are unlikely to control the pandemic.

The tissue receptor for SARS-CoV-2 is angiotensin converting enzyme 2 (ACE2), also the receptor for SARS-CoV-1 that emerged in 2003 [3]. Infection with the SARS-CoV-2 down regulates ACE2 [4]. In an animal model, higher levels of ACE2 were associated with a reduction in the severity of acute respiratory disease syndrome (ARDS) [5]. Severe COVID-19 is frequently complicated by coagulation 
abnormalities, cardiovascular involvement, and acute renal failure [6] These complications reflect underlying endothelial dysfunction.

\section{COVID-19: vaccination and antiviral treatment}

Vaccines for COVID-19 will take at least 12 to 18 months to develop and many practical obstacles will have to be overcome for them to become widely available. Similarly, there are no licensed antiviral treatments for COVID-19. The World Health Organization (WHO) is conducting a 'megatrial' to test four broad-spectrum antiviral treatments. Among them, lopinavir and ritonavir (a protease inhibitor combination used to treat HIV patients) and hydroxychloroquine have already been shown not to be effective in hospitalized COVID-19 patients. A randomized controlled trial in the United States (US) showed that remdesivir shortened the length of hospital stay by almost four days, but failed to significantly improve survival [7]. Remdesivir has recently been licensed to treat COVID-19 patients in the US, although a five-day course of intravenous treatment is expensive. Convalescent plasma is safe to use in COVID-19 patients, but implementing this treatment will be cumbersome and it is unlikely to be widely used [8]. Finally, dexamethasone, an inexpensive anti-inflammatory corticosteroid that has been extensively studied for treating sepsis and ARDS (with mixed results), improved survival in COVID-19 patients who required oxygen therapy [9].

Except for corticosteroids and anticoagulants, almost all of the agents (drugs, convalescent plasma, and monoclonal antibody preparations) that are or will be entered into clinical trials target the SARS-CoV-2 virus. Yet antiviral treatments might not be life-saving because most COVID-19 patients die in the second week of illness when virus titers have declined from peak levels observed earlier in the course of illness [10]. Instead of the virus, it is the host response that kills these patients.

In the absence of a 'game changing' treatment, uncertainty about the mortality risk for those who become infected is driving the global response to COVID-19. Individuals are asking "will I live or will I die?" They don't know the answer and neither do scientists or governments.

\section{COVID-19: treating the host response}

Statins and ARBs up regulate ACE2 and prevent experimental ARDS [2]. Together with clinical studies, these findings suggest that these drugs might reduce COVID-19 morbidity and mortality [11]. Several investigators have found that in hypertensive patients who were taking an ARB or an ACEI before or during COVID-19 hospitalization, treatment did not make the infection worse and, in some instances, was associated with improved survival [12-14]. Statins also improved survival in hospitalized COVID-19 patients [15]. 
Drugs like statins, ACEIs and ARBs (and other generic agents) counteract endothelial dysfunction [2]. They downregulate many of the pro-inflammatory cytokines (such as IL-6) associated with the 'cytokine storm'. They counteract coagulation abnormalities, bradykinin's effects on pulmonary vascular permeability (2, see Table 1), and promote mitochondrial biogenesis. They have broad effects on immunometabolism, are often involved in the resolution of inflammation, and probably promote greater tolerance of infection [2]. Greater tolerance might explain why children had lower mortality than adults during the influenza pandemic in 1918 [16], something also seen with COVID-19. These drugs won't cure the disease, but they might buy enough time to allow patients to recover on their own.

ARBs, ACEIs and statins have been shown to be safe in sepsis patients and they are well-tolerated in those with COVID-19. Although some clinicians and researchers have raised questions about the safety of these drugs, in COVID-19 patients, experts agree they should not be discontinued [12].

\section{Research on host response treatment}

Table 1 shows a research agenda for host response treatment of COVID-19 and other pandemic diseases. If studies such as these are not completed before the emergence of the next pandemic virus (such as a new influenza virus or coronavirus), they will surely be undertaken during the next pandemic's early days. Before the emergence of SARS-CoV-2, these studies were not even considered. Although many of them have been undertaken in recent months, the results of several observational studies appear to have had little practical impact on clinical care. Those that are randomized controlled trials will not produce results until sometime next year.

If effective, host response treatment could make an enormous contribution to global health, global equity, and global security [2]. Given these benefits, it is unclear why an idea that is more than ten years old continues to be ignored. Behavioral scientists have convincingly shown that biases, social influences and herding instincts among scientists and health officials can be important obstacles to introducing new scientific ideas [17]. These factors can "distort the evolution of knowledge if scientists are reluctant to accept an alternative explanation for their observations" [17]. In practical terms, for elite scientists and their sponsoring institutions "the reputational and/or financial costs of doing so can be very large" [2]. In other words, new ideas threaten the power of the scientific and global health establishment.

\section{The need for political leadership}

In 2008, I wrote, “... we have two alternatives: we can either do (research on host response treatment) before the pandemic arrives and perhaps show that generic agents will not be useful, or we can do it after the pandemic has passed and perhaps discover that millions of people could have been saved. We can no longer avoid this 
choice" [18]. In the runup to the COVID-19 pandemic, scientists and health officials ignored this choice.

In 2013, the Assistant Secretary for Preparedness and Response in the US, together with the Directors of the Centers for Disease Control and Prevention and the National Institutes of Health, set forth several components of a research response to a public health emergency like a pandemic [19]. They called for developing generic study protocols, pre-funding research networks, and awarding just-in-time research contracts to an on-call 'ready reserve' of clinicians, scientists, and other experts who would undertake this research. These sensible suggestions could have been implemented before the COVID-19 pandemic. Unfortunately, this was not done.

An article written by Michael Ignatieff in 2007 helps us understand what needs to be done to make host response treatment a reality [20]. Ignatieff reflected on his earlier (he thought mistaken) decision to support the US invasion of Iraq in 2003 [20].

\begin{abstract}
"The trouble with academics ... is that they care more about whether ideas are interesting than whether they are true. Politicians live by ideas just as much as professional thinkers do, but they can't afford the luxury of entertaining ideas that are merely interesting. They have to work with the small number of ideas that happen to be true and the even smaller number that happen to be applicable to real life. In academic life, false ideas are merely false and useless ones can be fun to play with. In political life, false ideas can ruin the lives of millions and useless ones can waste precious resources. An intellectual's responsibility for his ideas is to follow their consequences wherever they may lead. A politician's responsibility is to master those consequences and prevent them from doing harm."
\end{abstract}

In the context of pandemic preparedness, Ignatieff places responsibility squarely where it belongs: in the hands of political leaders. They include not only those in national governments and international agencies but also those in international philanthropic and humanitarian non-governmental organizations. He reminds them of their responsibilities not to pursue false and useless ideas that can waste precious resources and ruin the lives of millions. Instead, he urges them to support the small number of ideas that can be realistically applied to the lives of people everywhere.

\title{
What we should do
}

Without a new approach to treating patients with COVID-19 and other emerging infectious diseases, we could be sleepwalking into repeating the experience of patients and physicians during the influenza pandemic in 1918 [21]. Physicians who have cared for physicians who have cared for COVID-19 patients already know the difficulties they will face during the next pandemic.

COVID-19 patients already know the difficulties they will face during the next pandemic. Unfortunately, political leaders who are responsible for pandemic preparedness are still not pursuing practical ideas that could help them with their tasks. 
We have no idea of what the global mortality of the COVID-19 pandemic will be. However, the 1918 influenza pandemic killed an estimated 50-100 million people worldwide. If a new, highly virulent and easily transmissible influenza virus emerges, it too will spread rapidly and overwhelm healthcare systems everywhere. It could be far worse than anything we will experience with COVID-19.

Most people in the world will eventually be infected with SARS-CoV-2. Many will remain asymptomatic and others will develop a mild-to-moderate self-limited illness. Although clinical management of hospitalized COVID-19 patients has improved with the use of remdesivir [7], dexamethasone [9], anti-coagulation [22] and better management of oxygenation, many still develop severe illness and die. If we had more effective treatment, many of them would probably survive. Effective treatment might reduce hospital and ICU admissions and demands for oxygen therapy and mechanical ventilation. As prophylaxis, it might prevent illness in healthcare workers. Given to 'test-positive' individuals before they develop symptoms or in the initial stages of illness, it might prevent the development of more severe illness or the chronic, persistent illness that has been seen in many survivors (the so-called 'long-haulers'). There might be less need for widespread testing, contact tracing and isolation. Uncertainty about the disease might be greatly reduced and with it the devastating social, economic and political consequences experienced everywhere.

There are debates about how to best determine what constitutes effective treatment for COVID-19. Some investigators insist that nothing less than randomized controlled trials will be required, a view that dominates most 'top down' thinking about developing medical countermeasures for the current and future pandemics [23]. Others have a more nuanced (that is, more realistic) view of what is required; they recognize the necessary trade-offs between learning and doing [24].

Given the failure of scientists and health officials to undertake clinical trials of potentially effective treatments during the pre-COVID-19 period, some practicing physicians might decide to treat all of their COVID-19 patients (not just those with hypertension) with drugs (such as statins, ACEIs and ARBs, alone or in combination) whose effectiveness most still regard as uncertain. They will do so knowing treatment is safe and hoping it will do some good. It will be essential for them to report their observations on the effectiveness of these treatments if they choose to use them [25].

The great advantage of treating the host response for the COVID-19 and any future pandemic is that these inexpensive generic drugs are produced in developing countries and are widely available. Practicing physicians use them every day in caring for patients with common cardiovascular and metabolic diseases. If they could be shown to be clinically effective, these repurposed drugs could become a central feature of a worldwide 'bottom up' approach to managing all pandemics. In addition, there is a distinct possibility that they could also be used as syndromic treatment for patients with everyday acute critical illnesses (such as community-acquired pneumonia, sepsis, seasonal influenza) [2]. The cost of treating each patient would be a few dollars.

For more than a decade, I have argued that a practical response to a pandemic threat could be based on treating the host response with inexpensive generic drugs 
that are familiar to practicing physicians and would be available on the first pandemic day in every country that has a basic healthcare system [2]. Laboratory and clinical studies suggest that several inexpensive generic drugs already meet these criteria. If we already knew that these drugs could save lives, they could be used in every country that is still affected by COVID-19.

There is no convincing evidence (and no guarantee) that inexpensive generic drugs would reduce COVID-19 morbidity and mortality. Observational studies and randomized controlled trials of host response treatment for COVID-19 and everyday acute critical illnesses could provide crucial support for this idea. We should make this research a central element of our response to the COVID-19 pandemic. It should be the foundation of our response to the next one.

\section{Compliance with ethical standards}

Conflict of interest The author states there is no conflict of interest.

\section{References}

1. Fonarow GC, Wright RS, Spencer FA, Fredrick PD, Dong W, Every N, et al. Effect of statin use within the first 24 hours of admission for acute myocardial infarction on early morbidity and mortality. Am J Cardiol. 2005;96:611-6.

2. Fedson DS. Treating the host response to emerging virus diseases: lesson learned from sepsis, pneumonia, influenza and Ebola. Ann Transl Med. 2016;4:421.

3. Kuba K, Imai Y, Rao S, Gao H, Guo F, Guan B, et al. A crucial role of angiotensin converting enzyme 2 (ACE2) in SARS coronavirus-induced lung injury. Nat Med. 2005;11:875-9. https://doi. org/10.1038/nm1267.

4. Imai Y, Kuba K, Rao S, Huan Y, Guo F, Guan B, et al. Angiotensin-converting enzyme 2 protects from severe acute lung failure. Nature. 2005;436:112-6. https://doi.org/10.1038/nature03712.

5. Wösten-van Asperen RM, Lutter R, Specht PA, Moll GN, van Woensel JB, van der Loos CM, et al. Acute respiratory distress syndrome leads to reduced ratio of ACE/ACE2 activities and is prevented by angiotensin-(1-7) or an angiotensin II receptor antagonist. J Pathol. 2011;25:618-27. https://doi. org/10.1002/path.2987.

6. Gustafson D, Raju S, Wu R, Ching C, Veitch S, Rathnakumar K, et al. Overcoming barriers: the endothelium as a linchpin of coronavirus disease 2019 pathogenesis? Arterioscler Thromb Vasc Biol. 2020;40:1818-29. https://doi.org/10.1161/ATVBAHA.120.314558.

7. Beigel JH, Tomashek KM, Dodd LE, Mehta AK, Zingman BS, Kalil AC, et al. Remdesivir for the treatment of Covid-19 - Final report. N Engl J Med. 2020; 383:1813-26. https://doi.org/10.1056/ NEJMoa2007764.

8. Xia X, Li K, Wu L, Wang Z, Zhu M, Huang B, et al. Improved clinical symptoms and mortality on severe/critical COVID-19 patients utilizing convalescent plasma transfusion. Blood. 2020;136:7559.;126:1671-81 doi: https://doi.org/10.1182/blood.2020007079.

9. RECOVERY Collaborative Group, Horby P, Lim WS, Emberson J, Mafham M, Bell J, et al. Dexamethasone in hospitalized patients with Covid-19: preliminary report. N Engl J Med. 2020. https:// doi.org/10.1056/NEJMoa2021436.

10. Wofel R, Corman VM, Guggemos W, Seilmaier M, Zange S, Müller MA, et al. Virological assessment of hospitalized patients with COVID-19. Nature. 2020;581:465-9. https://doi.org/10.1038/ s41586-020-2196-x.

11 Fedson DS, Opal SM, Rordam OM. Hiding in plain sight; an approach to treating patients with severe COVID-19 infection. mBio. 2020;11:e00398-e420. https://doi.org/10.1128/mBio.00398-20. 
12. South AM, Diz DI, Chappell MC. COVID-19, ACE2, and the cardiovascular consequences. Am J Physiol Heart Circ Physiol. 2020;318:H1084-90. https://doi.org/10.1152/ajpheart.00217.2020.

13. Zhang P, Zhu L, Cai J, Lei F, Qin JJ, Xie J, et al. Association of inpatient use of angiotensin converting enzyme inhibitors and angiotensin II receptor blockers with mortality among patients with hypertension hospitalized with COVID-19. Circ Res. 2020;126:1671-81. https://doi.org/10.1161/ CIRCRESAHA.120.317134.

14. Bean D, Kralijevic Z, Searle T, Bendayan R, O'Gallagher K, Pickles A, et al. Angiotensin-converting enzyme inhibitors and angiotensin II receptor blockers are not associated with severe COVID19 infection in a multi-site UK acute hospital trust. Eur J Heart Fail. 2020;22:967-74. https://doi. org/10.1002/ejhf.1924.

15. Zhang XJ, Qin JJ, Cheng X, Shen L, Zhao YC, Yuan Y, et al. In-hospital use of statins is associated with a reduced risk of mortality among individuals with COVID-19. Cell Metab. 2020;32:176-87. https://doi.org/10.1016/j.cmet.2020.06.015.

16. Fedson DS. Influenza, evolution, and the next pandemic. Evol Med Public Health. 2018;2018(1):260-9. https://doi.org/10.1093/emph/eoy027.

17. Baddeley M. Herding, social influences and behavioural bias in scientific research. EMBO Rep. 2015;16:902-5. https://doi.org/10.15252/embr.201540637.

18. Fedson DS. Confronting an influenza pandemic with inexpensive generic agents: can it be done? Lancet Infect Dis. 2008;8:571-6. https://doi.org/10.1016/S1473-3099(08)70070-7.

19. Lurie N, Manolio T, Patterson AP, Collins F, Frieden T. Research as a part of public health emergency response. N Engl J Med. 2013;368:1251-5. https://doi.org/10.1056/NEJMsb1209510.

20. Ignatieff M. Getting Iraq wrong. New York Times Sunday Magazine. August 5, (2007).

21 Starr I. Influenza in 1918: recollections of the epidemic in Philadelphia 1976. Ann Intern Med. 2006;145:138-40.

22. Thachil J, Juffermans NP, Ranucci M, Connors JM, Warkentin TE, Ortel TL, et al. ISTH DIC subcommittee communication on anticoagulation in COVID-19. J Thromb Haemos. 2020;18:2138-44. https://doi.org/10.1111/jth.15004.

23. Simpson S, Kaufmann MC, Glozman V, Chakrabarti A. Disease X: accelerating the development of medical countermeasures for the next pandemic. Lancet Infect Dis. 2020;20:e108-15. https://doi. org/10.1016/S1473-3099(20)30123-7.

24. Angus DC. Optimizing the trade-off between learning and doing in a pandemic. JAMA. 2020;323:1895-6. https://doi.org/10.1001/jama.2020.4984.

25. Vandenbroucke JP. Observational research, randomised trials, and two views of medical science. PLoS Med. 2008;5:e67. https://doi.org/10.1371/journal.pmed.0050067.

Publisher's Note Springer Nature remains neutral with regard to jurisdictional claims in published maps and institutional affiliations. 